\title{
Drag Prediction and Decomposition Based on CFD Computations*
}

\author{
Wataru YAMAZAKI**, Kisa MATSUSHIMA** and Kazuhiro NAKAHASHI**
}

\begin{abstract}
In this paper, advanced drag prediction methods based on the momentum conservation theorem were applied to CFD computational results. These methods can decompose total drag into drag components such as wave, viscous and induced drag as well as spurious drag attributed to numerical computations. Hence the more accurate drag prediction is possible by the elimination of the spurious drag term. The computational results showed that the advanced methods had the good capability of drag prediction and meaningful drag decomposition with accuracy. It was also found that the predicted physical drag values were weakly dependent on the mesh quality.
\end{abstract}

Key Words: Drag Prediction and Decomposition, Spurious Drag, Mesh Dependency, CFD

\section{Introduction}

As the most important parameters for the aerodynamic performance of an aircraft, the lift and drag in the cruising condition are well known. Recently, owing to the advances on numerical schemes and the rapid growth of the computer, Computational Fluid Dynamics (CFD) has achieved a significant progress. However, the accurate drag prediction in CFD is still a major challenge for researchers today $^{(1)}$. In fact, the accuracy of drag prediction within 1 count (1e-4, about $0.4 \%$ in total drag of typical aircraft) is not achieved yet today.

Traditionally, surface integration of the pressure and stress tensor on the body surface of an aircraft, which is called 'Near-Field Method', is used for the drag prediction in CFD computations. But it is pointed out that the drag computed by the near-field method has inaccuracy relating to the numerical errors. Therefore, two advanced drag prediction methods based on the momentum conservation theorem around an aircraft is watched with keen interests. One is called 'Wake Integration' or 'Far-Field Method'(2)-(4) which can compute drag components from the surface integration on the wake plane of the downstream of an aircraft. The other is called 'MidField Method'(5)-(7) which is a volume integral method derived from 'Far-Field Method' by applying the diver-

* Received 22nd November, 2004 (No. 04-4220)

** Department of Aeronautics and Space Engineering, Tohoku University, Aramaki-Aza-Aoba, 6-6-01 AobaWard, Sendai 980-8579, Japan.

E-mail: yamazaki@ad.mech.tohoku.ac.jp gence theorem of Gauss. It is expected that these methods will be able to remove the effect of numerical errors from the computed drag coefficient.

Another advantage of these advanced drag prediction methods is that they have the drag decomposition capabilities. The aerodynamic design and optimizations of aircraft using CFD are widely conducted in recent days. In these projects, the drag reduction level and the mechanisms have to be discussed in detail such as each drag component level. Thus, these methods can be an essential tool for aircraft designers.

In this paper, therefore, the advanced drag prediction methods based on the momentum conservation theorem were applied to CFD computational results and the capability was analyzed.

\section{Drag Prediction Method}

In this chapter, three drag prediction methods were introduced in short.

\subsection{Near-field method}

Drag force is computed by the near-field method as follows:

$$
D=\iint_{\text {Body }}\left[-\left(P-P_{\infty}\right) n_{x}+\vec{\tau}_{x} \cdot \vec{n}\right] d s
$$

where $P$ is pressure, $\tau$ is the stress tensor, $\vec{n}$ is the unit normal vector to the aircraft surface. $n_{x}$ is $x$-component of the vector $\vec{n}, x$ is the free stream flow direction. The integral area 'Body' indicates surface of aircraft. The first and second term are called pressure drag and skin friction drag term respectively. 


\subsection{Far-field method (Wake integration)}

It is known that the drag prediction formula based on the momentum conservation theorem is transformed as follows by the use of the small perturbation approximation $^{(2)}$ :

$$
D=\iint_{W A} P_{\infty} \frac{\Delta s}{R} d s+\iint_{W A} \frac{\rho_{\infty}}{2}\left(v^{2}+w^{2}\right) d s+O\left(\Delta^{2}\right)
$$

where $\Delta s$ is entropy production, $R$ is the gas constant, $\rho$ is density, $(u, v, w)$ is velocity, $\Delta$ is the perturbation term. Integral area ' $W A$ ' indicates a wake plane normal to free stream flow direction. The first term of Eq. (2) corresponds to entropy drag which including wave, viscous and spurious drag. On the other hand, the second term originates in the vorticity, that is corresponds to induced drag. This induced drag term, called kinetic energy form, can be transformed more accurate formula called Maskell's form as follows ${ }^{(2)}$ :

$$
D_{\text {induced }}=\iint_{W A} \frac{\rho_{\infty}}{2}\left(v^{2}+w^{2}\right) d s \approx \iint_{W A} \frac{\rho_{\infty}}{2} \psi \xi d s
$$

where $\xi$ is the $x$-component of a vorticity vector and $\psi$ is a scalar potential obtained from a Poisson equation $\partial^{2} \psi / \partial y^{2}+\partial^{2} \psi / \partial z^{2}=-\xi . \psi$ looks like a stream function on the WA plane which is parallel to the $y-z$ plane.

\subsection{Mid-field method}

As mentioned before, mid-field method is derived from the far-field method by applying the divergence theorem of Gauss. Here, the concept is explained in short using the entropy term as follows:

$$
D_{\text {Entropy }}=\iint_{W A} \overrightarrow{\boldsymbol{F}}_{(\Delta s)} \cdot \vec{n} d s=\iiint_{V} \nabla \cdot \overrightarrow{\boldsymbol{F}}_{(\Delta s)} d v
$$

where $\overrightarrow{\boldsymbol{F}}_{(\Delta s)}$ is entropy drag seed vector in the wake plane, integral volume ' $\boldsymbol{V}$ ' indicates the flow field around the aircraft. Here, further drag decomposition of the entropy term is possible by the domain decomposition of the flow field $\boldsymbol{V}$. Physically, entropy production in the flow field will occurred within the shock region $\left(\boldsymbol{V}_{\text {shock }}\right)$ or wake and boundary layer region $\left(\boldsymbol{V}_{\text {viscous }}\right)$. So the entropy production in remaining region $\left(\boldsymbol{V}_{\text {spurious }}\right)$ is considered as unphysical (spurious) phenomena. The schematic sketch of the domain decomposition of a flow field is shown in Fig. 1. Then Eq. (4) can be transformed as follows:

$$
\begin{aligned}
& D_{\text {Entropy }}=\iiint_{V} \nabla \cdot \overrightarrow{\boldsymbol{F}}_{(\Delta s)} d v=\iiint_{V} F_{d i v \Delta s} d v \\
& =\iiint_{V_{\text {shock }}} F_{d i v \Delta s} d v+\iiint_{V_{\text {viscous }}} F_{d i v \Delta s} d v+\iiint_{V_{\text {spurious }}} F_{d i v \Delta s} d v \\
& =D_{\text {wave }}+D_{\text {viscous }}+D_{\text {spurious }}
\end{aligned}
$$

where $F_{d i v \Delta s}$ is entropy drag seed in the flow field. $D_{\text {spurious }}$ is the spurious drag component due to numerical diffusion

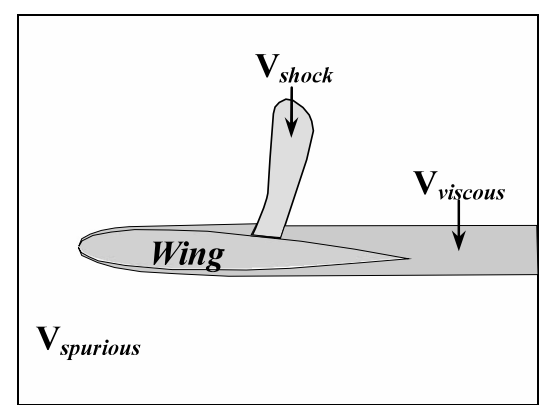

Fig. 1 Domain decomposition for mid-field method

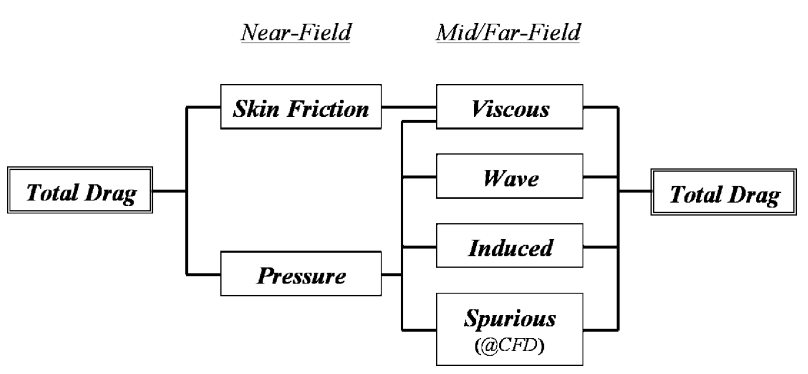

Fig. 2 Drag components and the classification

error. As readers guess, spurious drag may be generated in the shock and viscous region. But the spurious drag in these regions is insignificant, because the main part of the spurious drag generation occurred around the leading edge region which is outside of the viscous region. It will be shown later in this paper. Therefore, the advantage of the mid-field method is the capability to express the spurious drag term explicitly and to visualize entropy drag seed in the flow field.

The flow field is divided into by the use of following shock/boundary layer detective functions proposed by Ref. (7). In this research, for the detection of shock region, following function is adopted:

$$
f_{\text {shock }}=(\overrightarrow{\boldsymbol{V}} \cdot \nabla P) /(a|\nabla P|)
$$

where $\vec{V}$ is the velocity, $a$ is the local sonic speed. For the detection of viscous region, following function is adopted:

$$
f_{\text {viscous }}=\left(\mu_{l}+\mu_{t}\right) /\left(\mu_{l}\right)
$$

where $\mu_{l}$ and $\mu_{t}$ is laminar and eddy viscosity respectively.

\subsection{Summary of the drag components}

In Fig. 2, the correlation between each drag component which can be independently evaluated from CFD computational results is shown. The total drag is expressed as the sum of the pressure and skin friction drag using the near-field method. On the other hand, in the mid- and far-field method, the total drag is expressed as the sum of the viscous, wave, induced and spurious drag component.

\section{Flow Solver}

In this research, both of structured and unstructured mesh methods were used as flow solvers. For the structured mesh computation, two dimensional flows around 
an airfoil were analyzed. In these computations, compressible thin-layer Navier-Stokes (NS) equations were solved using C-type mesh. In space, the viscous diffusion terms were discretized by the central difference approximation. For the convection terms, the 3rd-order MUSCL type TVD scheme was used. In time, LU-SGS implicit method $^{(8)}$ was used for the time integration. The BaldwinLomax algebraic $\operatorname{model}^{(9)}$ was adopted to treat turbulent boundary layers.

For the unstructured mesh computation, three dimensional flows around wing geometry were analyzed. Compressible Euler equations were solved by a finite-volume cell-vertex scheme. The numerical flux normal to the control volume boundary was computed using an approximate Riemann solver of Harten-Lax-van-Leer-EinfeldsWada (HLLEW $)^{(8)}$. The second order spatial accuracy was realized by a linear reconstruction of the primitive gas dynamic variables inside the control volume with Venkatakrishnan's limiter ${ }^{(10)}$. LU-SGS implicit method for unstructured meshes ${ }^{(11)}$ was used for the time integration.

\section{Results and Discussions}

\subsection{Structured mesh computation}

Two dimensional viscous flow computation was conducted on structured meshes. A polar curve of NACA0012 airfoil was computed with the flow conditions which were the Mach number of 0.7 and the Reynolds number of 9.0e6. The mesh point was $303(200) \times 131$ (The number in parentheses is that of mesh points on the airfoil). The computational results are shown in Fig. 3 with experimental data ${ }^{(12)} . \boldsymbol{N F}$ means near-field computation, and $\boldsymbol{M F}$ is mid-field one. In this case, the induced drag component was not existed because the flow is two dimensional. The pressure contour map, domain decomposition map and entropy drag seed visualization in the flow field at three conditions are shown in Fig. 4.

Drag prediction based on the mid-field method showed good agreement with that of the near-field method and experimental data. The generation and increase of the wave drag $\left(\boldsymbol{M F} \_\boldsymbol{W A V E}\right)$ can be observed as the increase

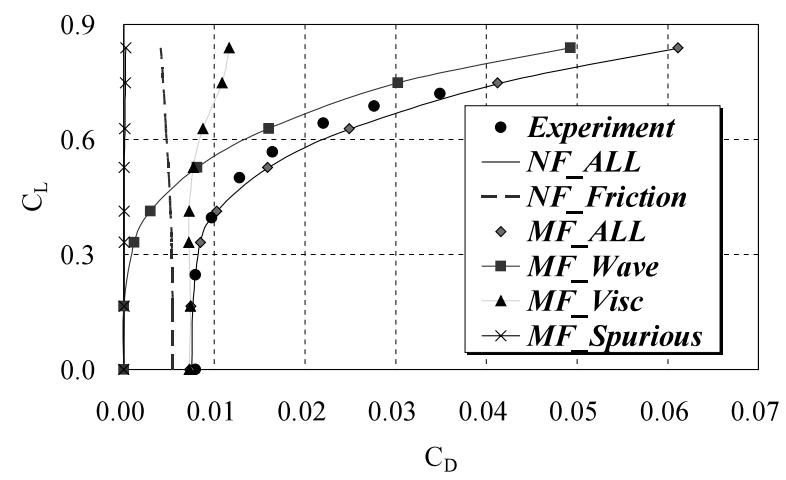

Fig. 3 Drag polar of NACA0012 @ Mach 0.7 of the angle of attack. The viscous drag term computed by the mid-field method ( $\left.\boldsymbol{M} \boldsymbol{F}_{-} \boldsymbol{V I S C}\right)$ overestimated that computed by the near-field method (NF Friction) especially in the high lift conditions. It is because the effect of the separation and increase of the wake thickness, which results in the increase of entropy production, is included in the viscous drag computation of the mid-field method. The spurious drag effect was little in this computation because of the adequate mesh quality.

For the investigation of the effect of the spurious drag depend on structured meshes, the mesh dependency study was conducted. Viscous flow computation around NACA0012 airfoil was conducted in the condition the Mach number 0.7, the angle of attack 2.5 degree. 4 meshes which have different mesh quality of resolution were prepared. The number of nodes of those meshes of level-1, $2,3,4$ is $163(60) \times 101,203(100) \times 131,303(200) \times 131$, $503(400) \times 131$ respectively. The drag prediction result is shown in Fig. 5.

MF PURE plot indicates the physical drag component, which is the result of removing spurious drag from

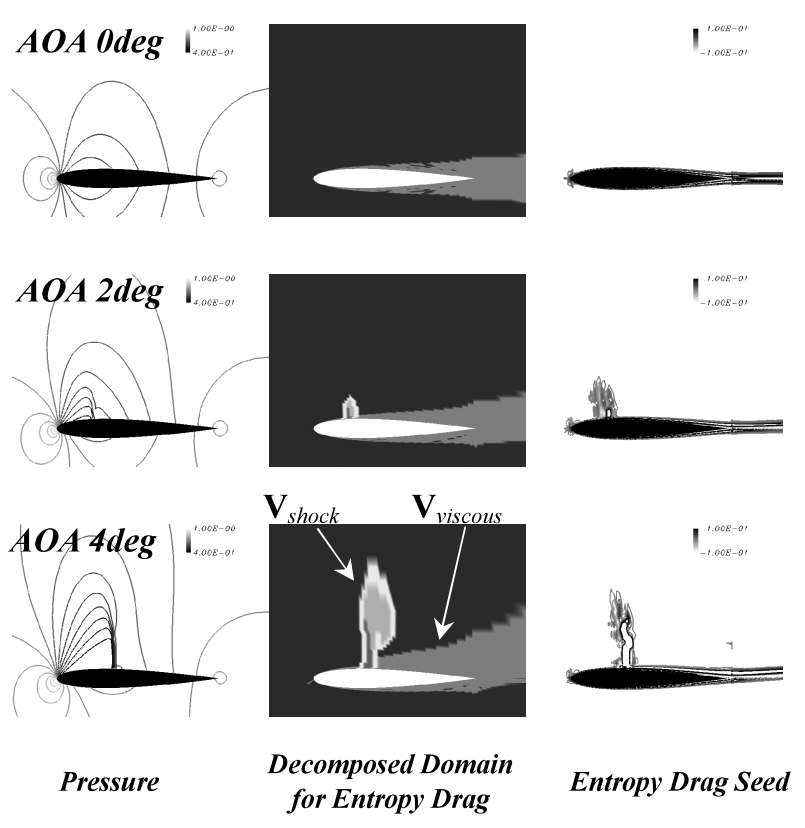

Fig. 4 Flow field visualization of the computation of drag polar

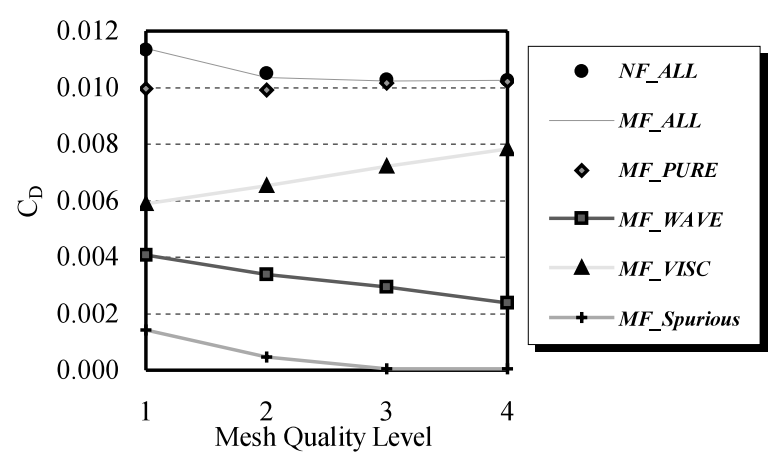

Fig. 5 Mesh dependency effect on structured meshes 
$\boldsymbol{M F} \boldsymbol{A} \boldsymbol{L} \boldsymbol{L}$ drag. As the increase of the mesh quality, the reduction of the total drag computed by the near-field method ( $\boldsymbol{N F} \boldsymbol{A} \boldsymbol{L L})$ was observed. It was confirmed that the reduction of the total drag computed by the mid-field method ( $\boldsymbol{M F} \boldsymbol{A} \boldsymbol{L} \boldsymbol{L})$ was also observed almost same level with the near-field plot. As the details, while the spurious drag was reduced rapidly with the increase of the mesh quality, PURE drag is almost constant. The difference of the drag value between the coarsest and finest mesh computation result was almost 1 count in the PURE drag, while it's 11 counts in the $\boldsymbol{N F} \boldsymbol{A} \boldsymbol{L} \boldsymbol{L}$ drag. In other words, with the mid-field method, it is possible to evaluate $\boldsymbol{P U R \boldsymbol { E }}$ drag without mesh dependency effect.

Flow field visualizations of the coarsest and finest meshes are shown in Fig. 6. Sharp shock wave was obtained with the finest mesh. With the coarsest mesh, unphysical entropy drag seed was observed around the leading edge. It was considered that this spurious drag production was caused by the numerical diffusions relating to the shortage of the mesh points against the rapid changes of flow variables around the leading edge. As the increase of the mesh quality, the increase of the viscous drag and the decrease of the wave drag were also observed. It is because the widen shock region steals viscous drag seeds on coarser meshes.

\subsection{Unstructured mesh computation}

Three dimensional inviscid flow computation was conducted on unstructured meshes. A polar curve of OneraM6 wing was computed in the flow condition the Mach number 0.84 and the range of the angle of attack is from -2 to 6 degree. The number of mesh points was 234621 . To evaluate the drag value, the combined midand far-field method was adopted. The entropy drag term was evaluated by the mid-field method and the induced drag term was evaluated by Maskell's form of the far-field method. The drag polar curve is shown in Fig. 7 and the visualization of $\lambda$-type shock wave is shown in Fig. 8.

The drag prediction by the combined mid- and far-

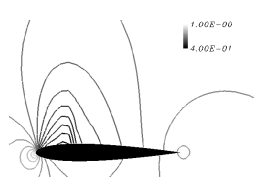

Level-1 (coarse)

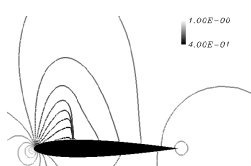

Level-4 (fine)

Pressure
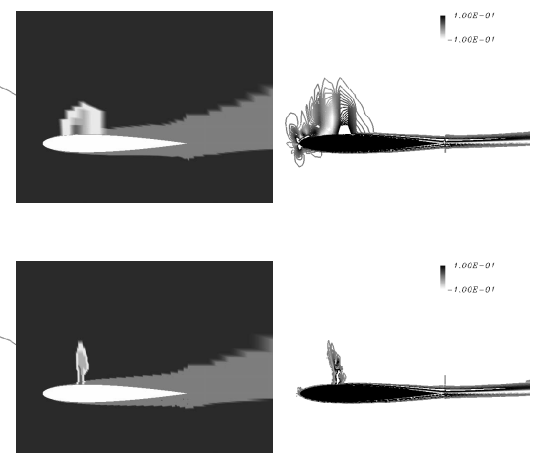

Decomposed Domain for Entropy Drag field method $(\boldsymbol{A} \boldsymbol{L} \boldsymbol{L})$ overestimates than the near-field drag prediction $(\boldsymbol{N F})$. It was considered by the analysis that the overestimation was due to the effect of the spurious drag term. It can be considered that the unstructured mesh computation has stronger effect of numerical diffusions than structured mesh computation. Therefore, a cutoff method which could extract the significant region for a physical parameter ${ }^{(4)}$ (here, it's corresponding to the entropy production) was used. In this cutoff method, by the trial and error study, a stable region of the spurious drag value which was almost insensitive to the small change of the cutoff value was determined. By the use of this method, spurious drag (Spurious_cutoff) was almost 30 counts at all conditions and the total drag (ALL_cutoff) showed good agreement with the near-field prediction. Entropy Drag plot is including the wave drag and spurious drag. As shown in Fig. 9, induced drag evaluated by the far-field method showed good agreement with the induced drag evaluated by the lifting line theory.

The visualization of the flow field at the $33 \%$ semispan station is shown in Fig. 10. Spurious drag seeds were widely observed around the leading and trailing edge. It is considered that this is because not only the shortage of the mesh resolution against the rapid changes of flow variables, but also the distortion of the unstructured cell elements around there.

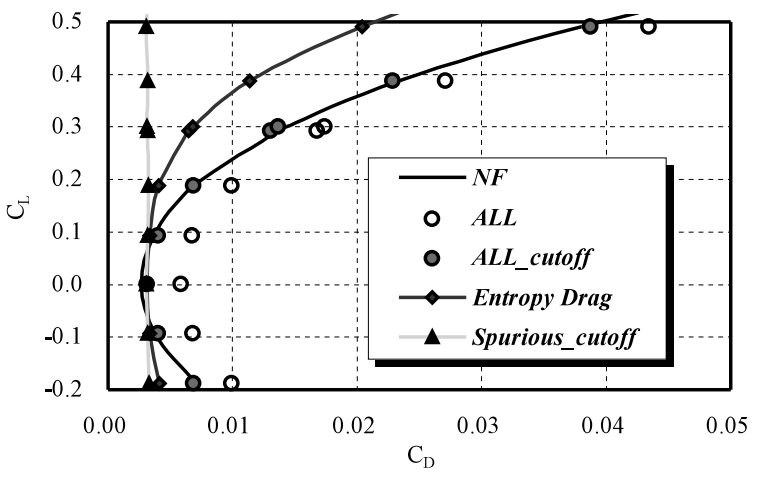

Fig. 7 Drag polar of OneraM6 @ Mach 0.84

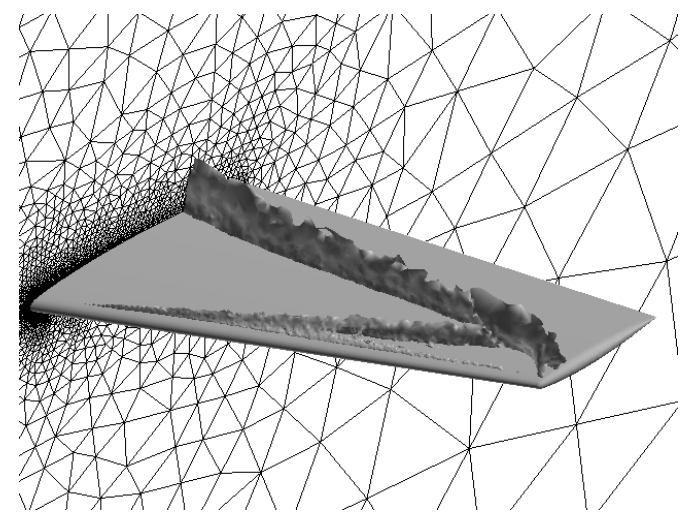

Fig. 8 Shock visualization of OneraM6 wing @ $M_{\infty}=0.84$, $\alpha=3.06$ degree

Fig. 6 Flow field visualization to show mesh dependency 


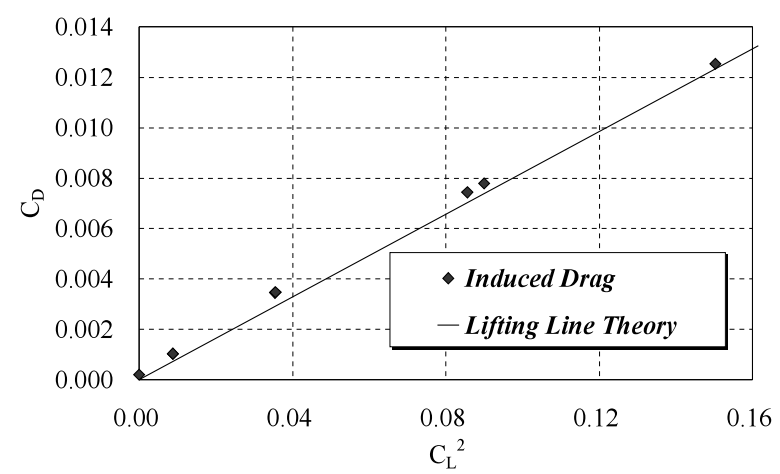

Fig. 9 Induced Drag- $C_{\mathrm{L}}^{2}$ plot
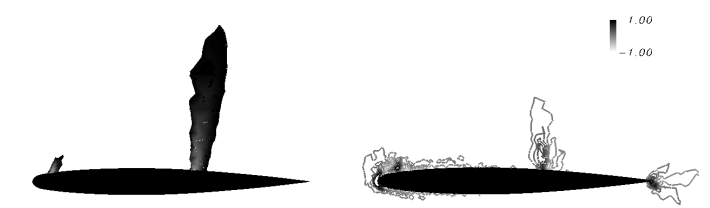

Shock Region Selection Entropy Drag Seed

Fig. 10 Flow field visualization at $33 \%$ semi-span

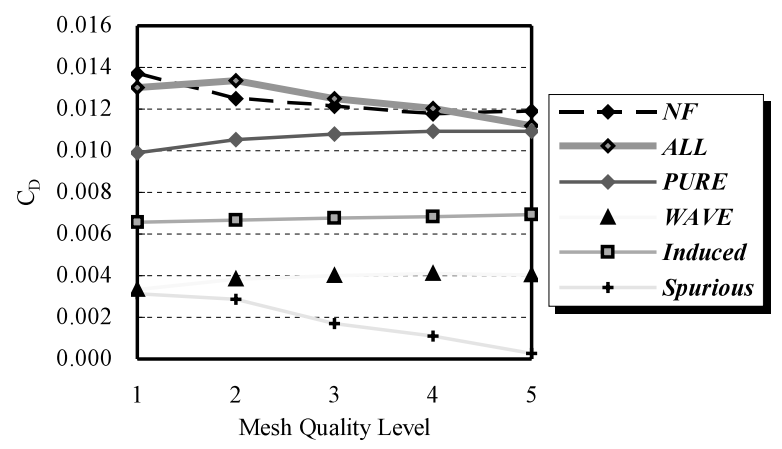

Fig. 11 Mesh dependency effect on unstructured meshes

The mesh dependency effect on unstructured meshes was also investigated. Inviscid flow computation around the OneraM6 wing was conducted in the flow condition the Mach number 0.84, the angle of attack 3.06 degree. 5 meshes which have different mesh quality level of resolution were prepared. The number of mesh points was from 200 thousands to 2.3 millions. Fine cells were arranged around the leading and trailing edge at finer meshes. The drag prediction results of various evaluation methods are displayed in Fig. 11. The Cp distributions at $44 \%$ semi-span are shown with experimental data ${ }^{(13)}$ in Fig. 12. Moreover, the mesh and entropy drag visualization of level-1, 3, and 5 is shown in Fig. 13. Similar to above result of drag polar, the entropy drag term was computed by the mid-field method and the induced drag was computed by the Maskell's form of the far-field method.

The results showed almost same tendency with that of structured mesh computation. As the increase of the mesh quality, the drag evaluated by the combined mid- and farfield method $(\boldsymbol{A L L})$ as well as the drag evaluated by the

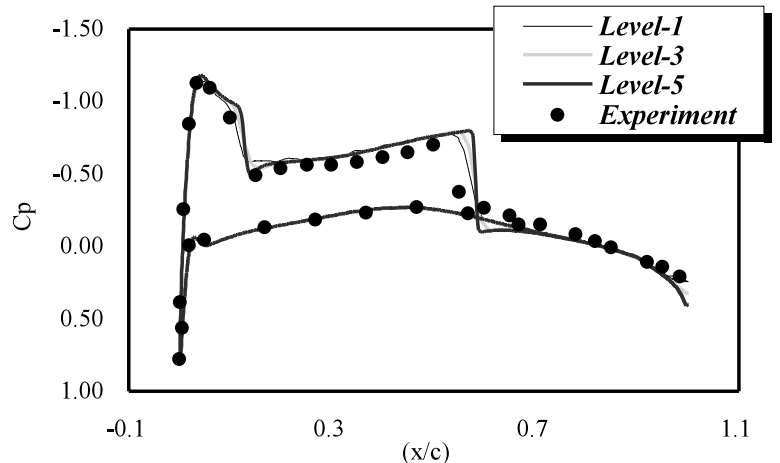

Fig. 12 Cp distribution at 44\% semi-span of OneraM6 wing
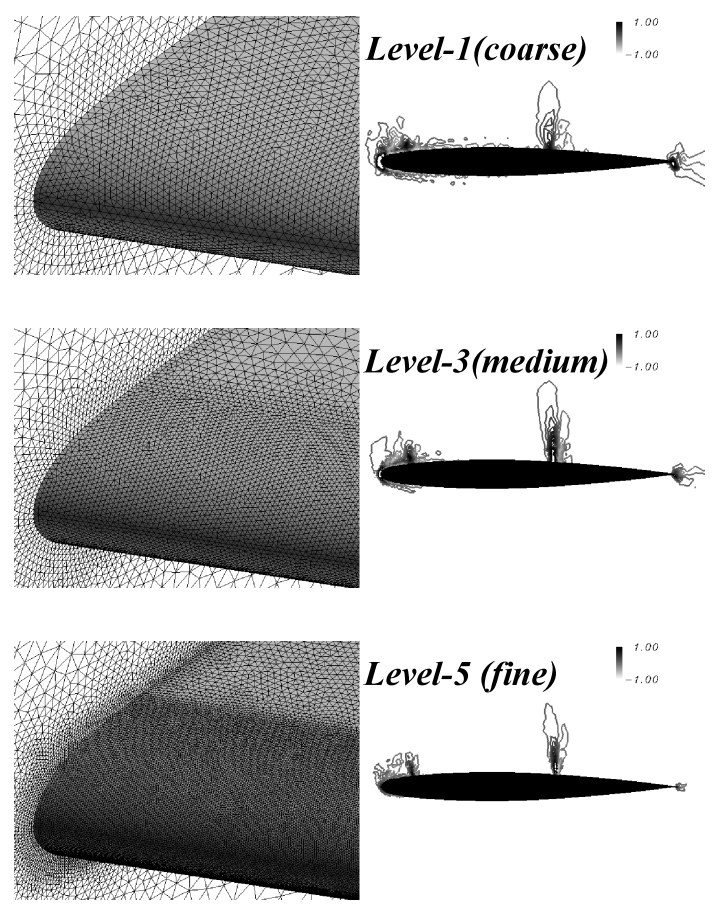

Mesh around the Leading Edge Entropy Drag Seed

Fig. 13 Mesh \& entropy drag seed visualization

near-field method $(\boldsymbol{N F})$ was reduced. On the other hand,

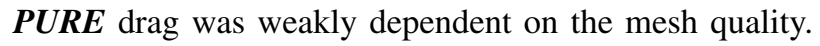
Wave drag as well as induced drag was also extracted weakly dependent on the mesh quality. The difference of the wave and induced drag value between the coarsest and finest mesh computation results was almost 7 and 3 counts respectively, while the difference was about 30 counts in the spurious drag. At the finest mesh, it was confirmed that shock wave was captured sharply and the spurious drag seeds around the leading and trailing edge were reduced.

\section{Conclusions}

In this paper, advanced drag prediction methods called 'Mid-Field Method' and 'Far-Field Method' were applied to CFD computational results. These methods had a capability of drag decomposition into physical drag components. Especially, the mid-field method could ex- 
tract the spurious drag component which originated in the effect of numerical errors. The far-field method with Maskell's form was useful for the induced drag evaluation.

The drag evaluated by the mid- and far-field methods showed good agreement with the drag evaluated by the near-field method and experimental data. By the investigation of the mesh dependency study, it was observed that the spurious drag reduced rapidly as the increase of the mesh quality of resolution. It was also confirmed that the physical drag components evaluated by the midand far-field methods were weakly dependent on the computational mesh quality. This confirmation and analysis could not be done without the mid- and far- field methods. We can evaluate each drag component separately with the methods, and can exclude the unphysical component to evaluate pure drag. As for unstructured mesh computations, it is generally said that the effect of spurious drag is larger than that of structured mesh. To confirm this, computations same as those done in section 4.1 will be conducted using an unstructured mesh method. Then the spurious error evaluated by the mid-field method will be compared with that of structured mesh computation. This comparison will clarify the difference between the results of structured and unstructured mesh computation.

These advanced methods are promising for the drag prediction of more complicated objects such as full shape geometry of aircraft. Moreover, these methods will be essential tools to analyze the mechanism of drag reduction as well as to diagnose the quality of computational meshes. Thus, we are going to utilize these methods for aerodynamic design and optimization.

\section{References}

( 1 ) Hemsch, M. and Morrison, J., Statistical Analysis of CFD Solutions from 2nd Drag Prediction Workshop,
AIAA Paper 2004-0556, (2004).

(2) Kusunose, K., Advanced Wake Integration Method for Experimental Drag Prediction, von Karman Institute Lecture Series 2003-2, (2003).

( 3 ) Cummings, R.M., Giles, M.B. and Shrinivas, G.N., Analysis of the Elements of Drag in ThreeDimensional Viscous and Inviscid Flows, AIAA Paper 96-2482-CP, (1996).

( 4 ) Hunt, D.L., Cummings, R.M. and Giles, M.B., Wake Integration for Three-Dimensional Flowfield Computations: Applications, Journal of Aircraft, Vol.36, No.2 (1999), pp.366-373.

( 5 ) Schmitt, V. and Destarac, D., Recent Progress in Drag Prediction and Reduction for Civil Transport Aircraft at ONERA, AIAA Paper 98-0137, (1998).

( 6 ) Esquieu, S., Aircraft Drag Extraction from Patched Grid Computations, AIAA Paper 2003-3659, (2003).

( 7 ) Paparone, L. and Tognaccini, R., Computational Fluid Dynamics-Based Drag Prediction and Decomposition, AIAA Journal, Vol.41, No.9 (2003), pp.1647-1657.

( 8 ) Obayashi, S. and Guruswamy, G.P., Convergence Acceleration of a Navier-Stokes Solver for Efficient Static Aeroelastic Computations, AIAA Journal, Vol.33, No.6 (1995), pp.1134-1141.

(9) Baldwin, B.S. and Lomax, H., Thin Layer Approximation and Algebraic Model for Separated Turbulent Flows, AIAA Paper 78-257, (1978).

(10) Venkatakrishnan, V., On the Accuracy of Limiters and Convergence to Steady State Solutions, AIAA Paper 93-0880, (1993).

(11) Sharov, D. and Nakahashi, K., Reordering of Hybrid Unstructured Grids for Lower-Upper Symmetric Gauss-Seidel Computations, AIAA Journal, Vol.36, No.3 (1998), pp.484-486.

(12) Holst, T.L., Viscous Transonic Airfoil Workshop Compendium of Results, Journal of Aircraft, Vol.25, No.12 (1988), pp.1073-1087.

(13) CFD Verification \& Validation Web Page, http://www.grc.nasa.gov/WWW/wind/valid/ 